\title{
Using SEM techniques for failure analysis of critical aeroengine components
}

\author{
J. M. Silva*, V. Infante** and A. S. Brito**
}

* Departamento de Ciências Aeroespaciais, Universidade da Beira Interior, Edifício II das Engenharias, 6201-001 Covilhã, Portugal

** Departamento de Engenharia Mecânica - Instituto Superior Técnico Av. Rovisco Pais, 1049-001 Lisboa - Portugal

Corresponding author: jmas@ubi.pt

Aeroengine components operate under particularly severe conditions, including the combination of both high mechanical stresses and elevated temperatures in chemically aggressive environments. These normal operational requirements will eventually promote both cyclic and time dependent failure mechanisms related with creep-fatigue damages [1]. Therefore, the use of adequate preventive inspection techniques is mandatory in order to maximize safety and reliability levels of these components during service. However, there are some occasional and unpredictable material failures which must be conveniently investigated in order to avoid future hazardous situations. Scanning electron microscopy (SEM) can play a major role in the context of evaluation of failed components, allowing the observation of fracture surfaces and other material's features which are essential to the identification of some particular failure mechanisms.

This paper presents two case studies involving the application of SEM techniques in the evaluation of damaged components belonging to different aeroengines and which are made of two types of nickel base superalloys: a compressor blade (case A) and a turbine disk (case B). Several SEM observations were used in the analysis of the fracture surfaces in order to identify crack nucleation sites and also crack propagation marks related with cyclic or time dependent mechanisms. Figure 1 presents two SEM images obtained for the fracture surface of the failed compressor blade (case A). Figure 1(a) allows to identify clear striation marks which are closely spaced and propagating on flat plateaus joined by shear steps, and the crack path (black arrow) follows a typical transgranular pattern. These are common features of cyclic dependent crack growth mechanisms due to high cycle fatigue, which was probably induced by a vibration condition sufficient to propagate cracking from initial defects formed at the leading edges of the blade. Figure 1(b) shows evidences of brittle fracture near the final fracture region typical of nickel base superaloys at moderate temperatures, as well as pronounced radial marks indicating the direction of crack propagation. In case B, SEM analysis of the failed component was used in order to identify the mechanisms of damage resulting from distinct load frequencies. Figure 2 was obtained for two distinct zones of the fracture surface of the component. Fig. 2(a) shows a typical intergranular crack propagation region resulting from a low frequency loading at high temperature $\left(\mathrm{f}=1 / 33 \mathrm{~Hz}\right.$ and $\left.\mathrm{T}=650^{\circ} \mathrm{C}\right)$. These conditions promote time-dependent mechanisms of damage leading to very irregular fracture surfaces with some visible marks of cavities and oxidation reactions (dark spots). In the other hand, Fig. 2(b) reveals a smoother fracture surface resulting from a higher loading frequency $(\mathrm{f}=5 \mathrm{~Hz})$. In this case, a transgranular crack propagation mode is predominant and cyclic dependent mechanisms assume the major contribution to the damage process. The influence of the loading frequency is well documented in literature for this kind of materials [2,3], and was confirmed in the present case via a SEM analysis. 
From the results of the two cases considered in this investigation, it is possible to conclude that SEM analysis is a valuable tool in the detection and identification of several degrading mechanisms affecting the integrity of aeroengine components allowing the application of preventive actions during both de design and maintenance stages in order to maximize the reliability and safety levels of aeronautical operations.

References

[1] Carter, T.; "Common Failures in Gas Turbine Engines"; Engineering Failure Analysis, 12; Elsevier; 2005; pp 237-247.

[2] Evans, W.J., Jones, J.P., Williams, S.; "The Interactions Between Fatigue, Creep and Environment Damage in Ti 6246 and Udimet 720Li"; International Journal of Fatigue, Vol. 27; Elsevier; 2005; pp 1473-1484.

[3] Tong J., Byrne, J., "Effects of frequency on fatigue crack growth at elevated temperature", Fat. Fract. Eng. Mater.and Structures, 22; Blackwell Science Ltd; 1999; pp 185-193.

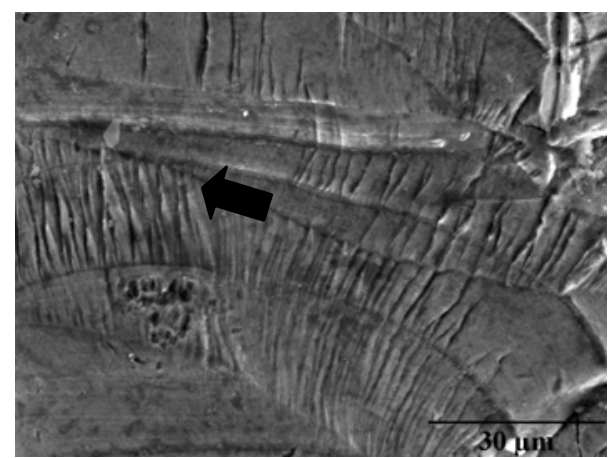

(a)

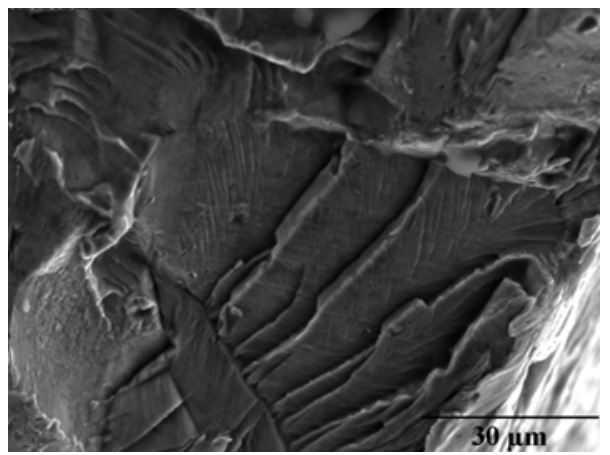

(b)

Fig.1 - SEM analysis of the fracture surface of a compressor blade; (a): striations due to cyclic plastic deformations related with fatigue mechanisms; (b): brittle fracture observed near the final fracture region.

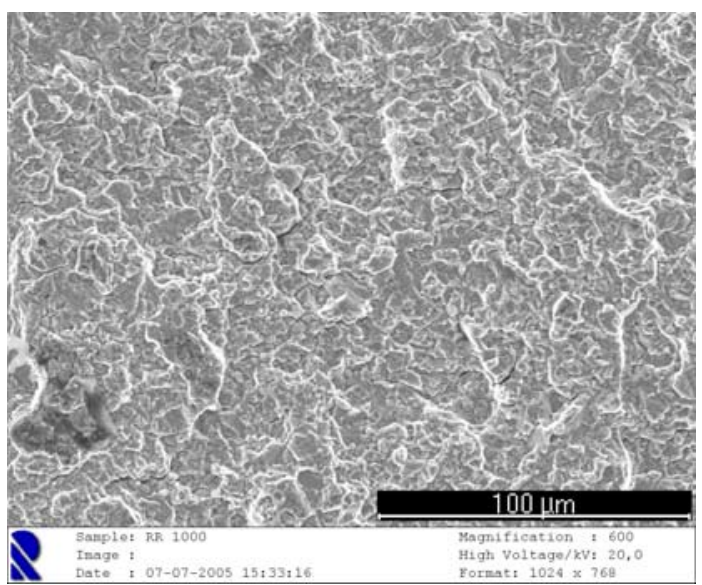

(a)

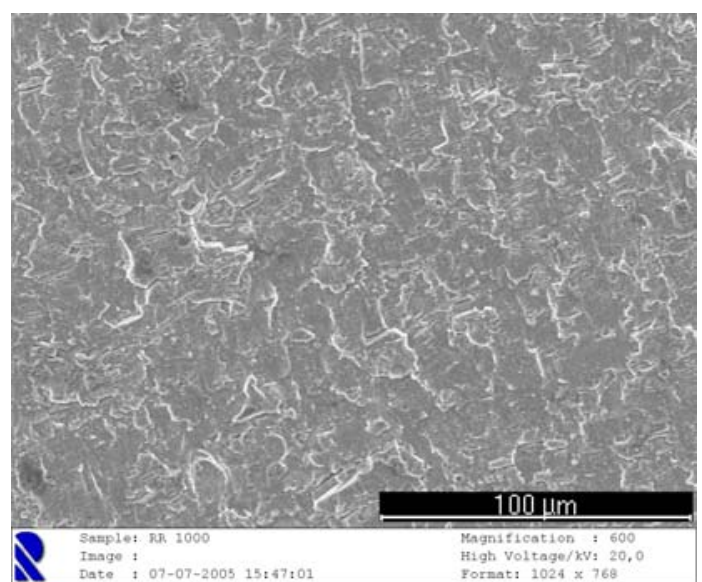

(b)

Fig. 2 - Identification of diferent crack propagation modes using SEM analysis of the fracture surface related with case B; (a): intergranular crack propagation; (b): transgranular crack propagation. 